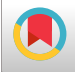

\title{
Explanation of the Factors Facilitating Self-Care Among Iranians' Veterans with Chronic Post-Traumatic Stress Disorder: A Qualitative
}

\section{Study}

Robabe Khalili ${ }^{1}$, Masoud Sirati Nir ${ }^{1,{ }^{*}}$, Masoud Fallahi Khoshknab (iD ${ }^{2}$, Hosein Mahmoudi ${ }^{3}$ and Abbas

Ebadi $^{1}$

${ }^{1}$ Behavioral Sciences Research Center, Life Style Institute, Nursing Faculty, Baqiyatallah University of Medical Sciences, Tehran, Iran

${ }^{2}$ Department of Nursing, University of Social Welfare and Rehabilitation Sciences, Tehran, Iran

${ }^{3}$ Trauma Research Center, Faculty of Nursing, Baqiyatallah University of Medical Sciences, Tehran, Iran

"Corresponding author: Associated Professor, Behavioral Sciences Research Center, Nursing Faculty, Baqiyatallah University of Medical Sciences, Tehran, Iran. Tel/Fax: +98-22218639, Email: masoudsirati@gmail.com

Received 2017 May 22; Revised 2017 July 18; Accepted 2018 January 18.

\begin{abstract}
Background: The lack of self-care in patients with post-traumatic stress disorder can cause physical health problems, which consequently result in a poor quality of life.

Objectives: This study aimed at identifying and exploring the factors facilitating self-care in veterans with chronic post-traumatic disorder.

Methods: This qualitative research was conducted by conventional content analysis. 12 veterans with the chronic post-traumatic stress disorder and 13 caregivers were selected using purposive sampling to participate in the study. Data collection was carried out during 18 individual interviews and two group interviews, which were profound and semi-structured.

Results: Five main categories including spirituality, personal knowledge, family support, social support, and institutional facilities were extracted as factors facilitating self-care in physical, psychological, emotional, and spiritual domains. A total of 14 subcategories emerged.

Conclusions: Planning and intervention on the factors facilitating self-care in different domains can help to achieve independence in caring for veterans with post-traumatic stress disorder.
\end{abstract}

Keywords: Caregivers, Chronic Post-Traumatic Stress Disorder, Self-Care, Qualitative Research, Veterans

\section{Background}

The Iraq-Iran war as one of the longest wars in the twentieth century in the Middle East (1980 - 1988) imposed psychological pressures and irreversible injuries, such as posttraumatic stress disorder (PTSD) on the Iranians veterans (1). PTSD along with other types of injury has been observed among Iranian combatants (2) so that $80 \%$ of the Iran-Iraq war veterans were diagnosed with this disorder (3). The prevalence rate of PTSD was reported to be $30 \%$ in soldiers in Vietnam (Koenen et al. 2008) and 14.9\% in Iran's military personnel (4).

According to research, those with experiences in dealing with traumatic events are impaired in their executive functions as they lack the ability to work independently, to carry out self-care activities, and to maintain their interpersonal relationships (5). Though PTSD itself is health threatening, the health is also highly threatened with inappropriate or unsuitable healthy habits and high-risk behaviors, by which the symptoms of PTSD further worsen (6).
The lack of self-care in patients with PTSD can cause physical health problems, which consequently lead to a poor quality of life (QOL) (7).

Self-care, as defined by the World Health Organization (WHO), is the ability of individuals, families, and communities to promote health, prevent disease, maintain health, and cope with illness and disability, with or without the support of a health care provider (8). Self-care in physical, psychological, emotional, and spiritual domains helps to restore the lifestyle balance in the PTSD patients so it can be effective in the recovery from PTSD (9). Most warriors do not fully understand the relationship between their combat experiences and their health conditions, and this issue applies especially to the stress and the long-term exposure to war experiences. Nurses play a key role in reducing problems associated with mental health-related stigma and provide support during the period of adaptation, training, and caring for fighting person (10). The use of nursing patterns in providing nursing care also improves the qual- 
ity of care and ultimately the patients' quality of life (11). Orem is the first nursing expert who postulated his theory in 1959, titled 'self-care nursing model', which is based on the ability of individuals and their need for self-care (12).

Some international studies have indirectly addressed the factors facilitating self-care among war troops. For example, Sayer et al. reported that the recognition and acceptance of the disease, the availability of help, having beliefs encouraging treatment and care, and organizational facilities such as a procedure to reduce stigma, improve access, screen for patients with PTSD, and encourage and facilitate social networks are factors facilitating the treatment among veterans with PTSD (13). Some studies in Iran have investigated the factors facilitating self-care of medical and surgical chronic patients. Thus far, no study has been directly conducted in Iran on the factors facilitating self-care in PTSD patients engaging in the Iran-Iraq war. Since multiple factors such as race, culture, values, and beliefs influence the perception and experience of care by people and given a difference in the Iranian society with other societies in terms of culture and familial and social values, the necessity of this study was therefore recognized.

The phenomenon of self-care is also associated with humans and human values that have broad and complex dimensions and in this respect, the use of naturalistic research is beneficial because it focuses on the comprehensive and in-depth investigation of human experiences on the desired phenomenon.

\section{Objectives}

Due to the shortage of available information and the importance of self-care in veterans with chronic warinduced PTSD, this qualitative research was conducted to explain the factors facilitating self-care in them.

\section{Materials and Methods}

\subsection{Design}

This qualitative study was accomplished by the approach of conventional content analysis (14).

\subsection{Setting and Participants}

The participants included 12 patients with chronic PTSD from among Iran-Iraq war combatants and 13 caregivers (7 spouses and 6 clinicians) who were interviewed over a 16-month period. The participants were recruited by referral from colleagues. Thus, by using a purposive sampling (14), they were selected from Baqiyatallah Hospital, Sadr Psychiatric Clinic, and the counseling center of Tehran West. The inclusion criteria included Iran-Iraq war veterans diagnosed with PTSD and having the ability to communicate verbally, as well as all people involved in their care process. War veterans with PTSD who were diagnosed with psychotic hallucinations and personality disorders were excluded.

Sample Questions of Interviews

- How have you solved your problems of illness?

- How did you take care when your husband shows illness relapse?

- How have you seen the self-care of veterans with chronic PTSD?

\subsection{Data Collection}

Face-to-face, semi-structured individual interviews were employed for data collection. In total, 18 individual interviews were done with patients and clinicians. No participant was rejected or dropped out until the end of the research. Everyone was interviewed once. Two (six-person) group interviews were conducted only with seven spouses. The individual interviews lasted for 40 to 90 minutes while focused group interviews lasted for 120 minutes. Data saturation was achieved when extra coding was no longer possible. The focused group interviews were performed under the direction of the first author at the Counselling Centre of Tehran West. All interviews were conducted by the first author, simultaneously recorded with a digital recorder, and transcribed verbatim within 24 hours using the Word application. In fact, data analysis was continuously carried out concurrently with data collection. MAXQ software was applied to facilitate encoding and a more structured analysis of interviews.

\subsection{Data Analysis}

The data analysis was done in eight stages using content analysis (15). These stages included data preparation, semantic units' determination, text encoding, codes reviewing, categories development, categories reviewing, themes identification, and findings report (Table 1).

\subsection{Rigor}

The credibility of data (16) was increased via prolonged engagement with the subject matter, member check, comparative, and continuous analysis. Data reliability was ensured through a review by all authors, peer check, or faculty member check. For conformability, all stages and the research process were accurately recorded and reported. For data transferability, attempts were made to present the participants' quotations verbatim. 


\begin{tabular}{llll}
\hline Table 1. The Process of Analyzing the Main Category & Condensed Meaning Unit & Code & \multicolumn{1}{c}{$\begin{array}{l}\text { Subcategory } \\
\text { Category }\end{array}$} \\
\hline Meaning Unit & $\begin{array}{l}\text { The usefulness of university education in } \\
\text { I went to university; this education was } \\
\text { very useful in accepting my illness }\end{array}$ & $\begin{array}{l}\text { The usefulness of university } \\
\text { education }\end{array}$ & $\begin{array}{l}\text { Formal } \\
\text { education }\end{array}$ \\
$\begin{array}{llll}\text { I study a lot of psychology books so as to } \\
\text { know how to deal with my illness }\end{array}$ & $\begin{array}{l}\text { Studying psychology books to know how to } \\
\text { deal with illness }\end{array}$ & $\begin{array}{l}\text { Studying to make awareness } \\
\text { about the illness }\end{array}$ & $\begin{array}{l}\text { Individual } \\
\text { Studies }\end{array}$ \\
\hline
\end{tabular}

\subsection{Ethical Considerations}

This study was approved by the Research and Ethics Committee of Baqiyatallah University of Medical Sciences (IR.BMSU.REG.95225). Ethics in research, such as informed consent, maintenance of anonymity, and participants' authority to leave the study at any desired time, were observed.

\section{Results}

This study was conducted on 25 participants (Tables 2 4).

Based on the data analysis, the five main categories including spirituality, personal knowledge, family support, social support, and institutional facilities were derived as factors facilitating self-care in war veterans with PTSD (Table 5).

\subsection{Spirituality}

Veteran's spirituality consists of three subcategories: 'faith and religious/spiritual beliefs', 'morality and humanity', and 'commitment to the revolutionary values'. In fact, the spirituality of veterans suffering from PTSD gave rise

\begin{tabular}{lccc}
\hline \multicolumn{4}{l}{ Table 2. Demographic } \\
\hline Participant No. & Age & Education & Comorbidity Disorders \\
\hline $\mathbf{1}$ & 75 & Bachelor & - \\
$\mathbf{2}$ & 52 & Diploma & - \\
\hline $\mathbf{3}$ & 49 & Under diploma & Depression \\
$\mathbf{4}$ & 50 & Under diploma & Anxiety \\
\hline $\mathbf{5}$ & 49 & Under diploma & Anxiety \\
$\mathbf{6}$ & 55 & Under diploma & Depression \\
\hline $\mathbf{7}$ & 47 & Under diploma & Depression \\
\hline $\mathbf{8}$ & 44 & Doctoral & Depression \\
\hline $\mathbf{9}$ & 41 & Bachelor & Drug abuse \\
\hline $\mathbf{1 0}$ & 63 & Under diploma & Depression \\
\hline $\mathbf{1 1}$ & 50 & Diploma & Drug abuse \\
$\mathbf{1 2}$ & 57 & Associate Degree & Bipolar disorder \\
\hline
\end{tabular}

\begin{tabular}{|c|c|c|c|}
\hline Participant No. & Age & Education & $\begin{array}{l}\text { Place of Focused } \\
\text { Group Interview }\end{array}$ \\
\hline $\mathbf{1}$ & 45 & Diploma & \multirow{7}{*}{$\begin{array}{c}\text { The counseling center } \\
\text { of Tehran West }\end{array}$} \\
\hline 2 & 31 & Student of university & \\
\hline 3 & 48 & Diploma & \\
\hline 4 & 43 & Diploma & \\
\hline 5 & 49 & Bachelor & \\
\hline 6 & 50 & Under diploma & \\
\hline 7 & 52 & Diploma & \\
\hline \multicolumn{4}{|c|}{ Table 4. Demographic Characteristics of Clinicians } \\
\hline Participant No. & Age & Working Experience & Position \\
\hline 1 & 53 & 15 & Psychiatrist \\
\hline 2 & 53 & 16 & Psychiatrist \\
\hline 3 & 47 & 17 & Clinical psychologist \\
\hline 4 & 48 & 23 & Social worker \\
\hline 5 & 48 & 2 & General physician \\
\hline 6 & 57 & 15 & Psychiatric nurse \\
\hline
\end{tabular}

to a better tolerance to control his illness and facilitate his spiritual self-care:

"When someone with strong beliefs is quietly exposed to disease-generating issues, nothing would happen to him, or his symptoms would go away very soon."(clinician 5)

"I went for God, I stand for God, I said that I went for God and bear it for God, and God would help me."(Patient 7)

\subsection{Personal Knowledge}

'Personal knowledge' consists of two sub-categories: 'formal education' and 'individual study'. In fact, this category states that the knowledge is obtained from formal education and individual studies in the veterans with PTSD with the aim of creating awareness about the illness and how to take care of it and facilitate the psychological/physical self-care: 


\begin{tabular}{ll}
\hline Table 5. Categories and Subcategories \\
\hline Main Categories & Subcategories \\
\hline Spirituality & Faith and religious/spiritual belief \\
\hline Morality and humanity \\
\hline Commitment to revolutionary values \\
\hline Fersonal knowledge & Formal education \\
\hline Individual studies \\
\hline Pamily support & Participation of wife in caring \\
\hline Having a family supportive atmosphere \\
\hline Treatment staff and supportive care \\
\hline
\end{tabular}

"I read a lot of psychology books so as to know how to deal with my illness, I learned how to control my anger and violence, I agreed to adhere to treatment and every month to be visited by my psychiatrist, and I take my medication on time."(Patient 8)

\subsection{Family Support}

Family support consists of two sub-categories: 'the participation of wife in caring' and 'having a familysupportive atmosphere'. Based on the analysis of the interviews, the family is a source of support for their physical/psychological/emotional self-care, and is considered as a factor that facilitates self-care for veterans with PTSD due to the contribution and companionship features of wives in caring, as well as the supportive environment:

"I often go along with my husband for his visit, I usually spoke to the psychiatrist and asked for a recommendation on how to better take care of my husband."(spouses 7)

"In order to keep me away from this situation, my family goes the extra mile: they laugh or tell a joke or suggest a topic to distract my thoughts and mind from a certain topic. When they do so, I suddenly become happy, and this happiness remains with me for a few days."(Patient 2)
"The home atmosphere is not to be irritated; wife and children try to maintain peace at home and be patient against his anger and aggression and avoid quarreling with him."(Clinician 3)

\subsection{Social Support}

Social support consists of two sub-categories: 'visiting relatives or close friends' and 'the assistance and the empathy of friends and colleagues'. According to the analysis of interviews, social support, such as visiting and expressions of emotions and empathy of relatives, is useful to improve his condition and emotional/psychological self-care, as well as the assistance and the support of combatant's friends and colleagues in the workplace could largely improve disease-related problems:

"Social respect of these patients is very important in terms of people and officials to have supportive behavior towards them; when these patients become socially isolated and frustrated, they will lose the motivation of their recovery and care."(Clinician 1 )

"I usually call and greet my relatives, but they come in person due to the fact that I am sick and unable to go to their homes. Now that I have been hospitalized, some of them call me, and some others come to visit me. My spirit has greatly changed."(Patient 7)

\subsection{Organizational Facilities}

Organizational facilities derived as a factor facilitating self-care of veterans with PTSD consist of five subcategories: 'the ease of access to facilities', 'the material supply of caregivers', 'conducting family education courses', 'making a suitable environment for treatment and hospitalization' and 'treatment staff and supportive care':

"When I attended the family education courses and performed in accordance with our training, our relationship became a lot better, and he became less nervous."(Spouse 4)

"I was hospitalized many times, this ward has a good discipline and is peaceful, they give us medicine on time, feed us on time, clean our room, hold prayer, and consult us. Whenever I am admitted, I feel better."(Patient 12)

"If I respect the patient, I would build the trust on arrival, and then I could communicate with him. Otherwise, there will be no perfect relationship and I will not be able to take proper care of them and meet their educational needs"(Clinician 6)

\section{Discussion}

The findings of this study offer a view of the factors facilitating self-care for war-PTSD veterans, based on the ac- 
tual experiences of participants. In the view of veterans with chronic PTSD and their caregivers, spirituality plays a major role in tolerance of problems and it has paved the way for spiritual self-care. In fact, spiritual beliefs are considered as the most important resource in coping with stress (17). In support to our findings, Hasanovic and Pajevic found that moral and religious beliefs in combatants enabled them to better achieve control, and they lead to an effective mechanism to curb the abuse of alcohol and drugs (18). In addition, there was a tendency toward spiritual care among veterans so that Bonner et al. reported that approximately half of the sample with PTSD and depression was open to seeking help from a spiritual counselor (19).

Another concept found in this study was the knowledge of veterans' formal education and individual studies, which enabled them to effectively take care of symptoms and problems related to their disease. Of course, knowledge and seeking health-related information play an important role in the management of chronic diseases so that adequate information about the disease is an essential component to enable people to change their behavior, improve their lifestyles, and promote health (20). Sayer et al. also stated that the lack of knowledge and awareness is one of the barriers to care at the individual and social level, which is consistent with our results (13). One of the main strategies for enhancing patients' health literacy is to provide them with educational resources (21) to facilitate their care process.

Another factor facilitating self-care in the current study was family support for veterans, which became apparent in the spouse's participation in caring and the family's supportive atmosphere. Although PTSD has negative effects on the performance and quality of life of patients and family members (22), weak performance on the part of the family is accompanied by negative consequences of treatment (23). The family's involvement clinically helps in assessing and planning treatment, and it is often accompanied by an increase in mutual consent of patient and family (24). In support of our findings, Fredman et al. indicated that the wife's compliance and consistency with PTSD patients were significantly associated with improvements in their depression and symptoms in cognitive-behavioral therapy (25).

In our study, the veterans expressed social support as a visitation from relatives and the assistance and empathy of combatants' friends and colleagues, which were helpful in improving their conditions and care. Although some studies stated the effect of social support on the protection from the risk of developing PTSD after traumatic events, other studies also demonstrated the effects of social support as a risk factor for PTSD. Koenen et al. showed the dual effects of social support as a risk and protective factor regarding PTSD so that veterans with PTSD who had greater community involvement were more likely to recover from their illness. Conversely, veterans who received more negative community attitudes on return to their home were more likely to maintain PTSD (26). Given the importance of the source role in social support, Laffaye et al. showed that the highest level of social support and the minimum perceived stress was from combatants' friends (27), and their findings are the same as those obtained in our study.

In this study, the organizational facilities such as the ease of access to the therapeutic resources and facilities were determined as the insurance coverage of treatment and care services, the availability of veteran's healthmonitoring physician, and the home visits by veterans. Having access to treatment and caring facilities facilitates the self-care process of the chronic PTSD veterans since many patients with PTSD are often discouraged from continuing their treatment due to the distance and the high cost of treatment, which make the disease more complex (28). Another organizational facility was the material supply of caregivers. Given that the majority of veterans with mental disorders have livelihood and welfare problems, an increase in the benefits could be helpful in solving the economic problems of families and could eliminate one of the main concerns of veterans. Conducting family education courses was another organizational facility that had a large impact on the improvement of the veteran's family relationships and engaging the family, especially his wife, in the caring. The importance of family involvement in the care of psychiatric disorders, particularly PTSD, has drawn increasing attention during the past two decades in America so that the family support and education program (SAFE) are included in the PTSD practical guidelines (29). In addition, a safe hospitalization environment and highly qualified medical and caring staff, which are concerned with the therapeutic communication and provide the appropriate medical, caring, and educational needs of veterans with PTSD, were influential in facilitating selfcare. It is important to note that combatants as a cultural group encounter special challenges with their health care provider due to the impact of culture on the healthcare performance. Thus, it is essential to determine effective responses to the unique healthcare needs of this group (30). A limitation to this study was the psychological condition of the patients, which might affect the interview process and their accountability that, in this case, it was tried to control a friendly atmosphere and to restore their confidence. 


\subsection{Conclusion}

Based on the finding, spirituality mediates the use of positive coping strategies. Thus, the integration of spiritual counselors into the care teams of veterans may be effective. The knowledge and the awareness of veterans led to effectively taking care of the symptoms and problems related to their disease. Therefore, educational planning on the principles of self-care can be effective steps to achieve independence in caring for veterans. In addition, according to the emerging importance of the family in facilitating self-care in the PTSD veteran, the therapeutic and care teams can focus more on family-centered interventions and resolve the problems in their family relationships. Social support through connectedness to social networks such as combatant's friend, relatives, and colleagues was found as a facilitator of self-care. Veterans can improve their perceived social support through participation in the rational social network and receiving positive community attitudes. The educational, therapeutic, and welfare facilities of the institution were influential in facilitating the self-care of veterans. The veterans, as a cultural group, have the unique healthcare needs; thus, we should plan for the establishment of their unique facilities.

\section{Acknowledgments}

This manuscript was extracted from a Ph.D. thesis. The authors would like to thank the authorities of the Foundation of Martyrs and Veterans Affairs in Great Tehran, as well as the medical and care staff of the psychiatric ward in Baqiyatallah Hospital, Sadr Psychiatric Clinic, Counselling Centre of Tehran West, and all participants of this study.

\section{Footnotes}

Authors' Contribution: Robabe Khalili accomplished the study design, data collection, and writing of the first draft. Masoud Sirati Nir contributed to the supervision of all the steps of the research. Additionally, Masoud Fallahi Khoshknab, Hosein Mahmoudi, and Abbas Ebadi equally assisted in reviewing, critical revision, and editing of the manuscript.

Declaration of Interests: None declared.

Funding/Support: The funding support of this research was done by Behavioral Sciences Research center and Nursing Faculty, Baqiyatallah University of Medical Sciences.

\section{References}

1. Mandani B, Javadipour S, Fakhri A. [The relationship between quality of life and percentage of injury among posttraumatic stress disorder veterans]. Health. 2013;5(3):183-92. Persian.
2. Mirzaei J. [Comparison of severity and prevalence post traumatic stress disorder in veterans with mental and physical complication from war][Dissertation]. Iran: Islamic Azad University Branch of Roodehen; 2004. Persian.

3. Bahreinian A, Borhani H. [Mental health in group of war veterans and their spouses in Qom]. Res Med. 2003;27(4):305-12. Persian.

4. Donyavi V, Shafighi F, Rouhani SM, Hosseini SR, Kazemi J, Arghanoun $S$, et al. The prevalence of ptsd in conscript and official staff of earth force in tehran during 2005-6. J Ann Mil Health Sci Res. 2007;5(1):1121-5. Persian.

5. Walter $\mathrm{KH}$, Palmieri PA, Gunstad J. More than symptom reduction: changes in executive function over the course of PTSD treatment. J Trauma Stress. 2010;23(2):292-5. doi: 10.1002/jts.20506. [PubMed: 20419740].

6. Jongsma Jr AE, Peterson LM, Bruce TJ. The complete adult psychotherapy treatment planner: includes DSM-5 updates. John Wiley \& Sons; 2014.

7. Ouimette P, Cronkite R, Henson BR, Prins A, Gima K, Moos RH. Posttraumatic stress disorder and health status among female and male medical patients. J Trauma Stress. 2004;17(1):1-9. doi: 10.1023/B:JOTS.0000014670.68240.38. [PubMed: 15027787].

8. Webber D, Guo Z, Mann S. Self-care in health: we can define it, but should we also measure it. Self Care. 2013;4(5):101-5.

9. The National Center for PTSD. Self-care following trauma - hidden hurt. 2015. Available from: Http://www.hiddenhurt.co.uk/self-care following_trauma.html.

10. Algire M, Martyn D. Enhancing emergency nurses' knowledge of veterans' health needs. J Emerg Nurs. 2013;39(6):570-5. doi: 10.1016/j.jen.2013.08.002. [PubMed: 24209587].

11. Malekzadeh J, Amouzeshi Z, Mazlom R. [The effect of teaching Orem self-care model on nursing students communication performance in clinical setting]. Modern Care J Abnormal Psychol. 2011;8(3):107-15. Persian.

12. Ghanbari A, Parsaykta Z, Faqieh Zadeh S, Sadat Hashemi S. [Application of Self-Care Model in determining the factors affecting on quality of life and metabolic status of non-insulin dependent diabetic patients using path analysis]. Daneshvar Med Sci Res J Shahed Univ. 2004;11(51):65-74. Persian.

13. Sayer NA, Friedemann-Sanchez G, Spoont M, Murdoch M, Parker LE, Chiros C, et al. A qualitative study of determinants of PTSD treatment initiation in veterans. Psychiatr Interpers Biol Proc. 2009;72(3):238-55. doi: 10.1521/psyc.2009.72.3.238.

14. Holloway I, Galvin K. Qualitative research in nursing and healthcare. John Wiley \& Sons; 2016.

15. Hsieh HF, Shannon SE. Three approaches to qualitative content analysis. Qual Health Res. 2005;15(9):1277-88. doi: 10.1177/1049732305276687. [PubMed: 16204405].

16. Lincoln YS, Guba EG. Naturalistic inquiry. Sage; 1985.

17. Drescher KD, Foy DW. Spirituality and trauma treatment: Suggestions for including spirituality as a coping resource. Nat Center PTSD Clin Q. 1995;5(1):4-5.

18. Hasanovic M, Pajevic I. Religious moral beliefs as mental health protective factor of war veterans suffering from PTSD, depressiveness, anxiety, tobacco and alcohol abuse in comorbidity. Psychiatr Danub. 2010;22(2):203-10. [PubMed: 20562748].

19. Bonner LM, Lanto AB, Bolkan C, Watson GS, Campbell DG, Chaney EF, et al. Help-seeking from clergy and spiritual counselors among veterans with depression and PTSD in primary care. J Religion Health. 2013;52(3):707-18. doi: 10.1007/s10943-012-9671-0.

20. Speros C. Health literacy: concept analysis.JAdv Nurs. 2005;50(6):63340. doi: 10.1111/j.1365-2648.2005.03448.x. [PubMed: 15926968].

21. Williams MV, Davis T, Parker RM, Weiss BD. The role of health literacy in patient-physician communication. Fam Med. 2002;34(5):383-9. [PubMed: 12038721]. 
22. Monson CM, Taft CT, Fredman SJ. Military-related PTSD and intimate relationships: from description to theory-driven research and intervention development. Clin Psychol Rev. 2009;29(8):707-14. doi: 10.1016/j.cpr.2009.09.002. [PubMed: 19781836]. [PubMed Central: PMC2783889].

23. Tarrier N, Sommerfield C, Pilgrim H. Relatives' expressed emotion (EE) and PTSD treatment outcome. Psychological Medicine. 1999;29(4):80111. doi: $10.1017 /$ s0033291799008569.

24. Prince JD. Family involvement and satisfaction with community mental health care of individuals with schizophrenia. Community Ment Health J. 2005;41(4):419-30. [PubMed:16335351].

25. Fredman SJ, Pukay-Martin ND, Macdonald A, Wagner AC, Vorstenbosch V, Monson CM. Partner accommodation moderates treatment outcomes for couple therapy for posttraumatic stress disorder.J Consult Clin Psychol. 2016;84(1):79-87. doi: 10.1037/ccp0000061. [PubMed 26501498]. [PubMed Central: PMC4696059].

26. Koenen KC, Stellman JM, Stellman SD, Sommer JF. Risk factors for course of posttraumatic stress disorder among vietnam veterans: A 14-year follow-up of american legionnaires. J Consult Clin Psychol. 2003;71(6):980-6. doi:10.1037/0022-006x.71.6.980.

27. Laffaye C, Cavella S, Drescher K, Rosen C. Relationships among PTSD symptoms, social support, and support source in veterans with chronic PTSD. J Trauma Stress. 2008;21(4):394-401. doi: $10.1002 /$ jts.20348.

28. Morland LA, Raab M, Mackintosh MA, Rosen CS, Dismuke CE, Greene CJ, et al. Telemedicine: A cost-reducing means of delivering psychotherapy to rural combat veterans with PTSD. Telemed e-Health. 2013;19(10):754-9. doi:10.1089/tmj.2012.0298.

29. Foa EB, Keane TM, Friedman MJ, Cohen JA. Effective treatments for PTSD: practice guidelines from the international society for traumatic stress studies. Guilford Press; 2008.

30. Hobbs K. Reflections on the culture of veterans. AAOHN J. 2008;56(8):337-41. doi: 10.3928/08910162-20080801-07. 